\title{
Myceleal Emphysematous Cystitis Complicating a Renal Transplant Kidney
}

\author{
Erich K. Lang, Ernest Rudman, Karl Zhang, Daniel Thorner, Amer Hanano \\ Department Radiology and Urology, SUNY Downstate Medical School, Brooklyn, New York, NY, \\ USA
}

This 44 year old Caucasian male presented acutely ill with chills, fever and pain over the renal allograft. The symptoms had intensified over the past 3 days. At admission his temperature was 100.8 F, pulse-rate 110/min, BP 185/110, Hb 10.2, Hct 28, WBC 16200 (66\% polymorphs). BUN $140 \mathrm{mg} / \mathrm{dL}$, CR $3.8 \mathrm{mg} / \mathrm{dL}$, blood sugar $121 \mathrm{mg} \%$.

The patient had a history of poorly controlled hypertension for eight years and received a renal transplant for endstage renal disease some 10 months ago. The postoperative period was complicated by several episodes of LUTIs which were treated with amoxicillin and ciprofloxacin. Two months prior to this admission the patient had been treated for acute rejection and was still on maintenance immunosuppressive therapy. Foley catheterization yielded only $100 \mathrm{~mL}$ of purulent urine; analysis revealing 60 $\mathrm{WBC} / \mathrm{hpf}$, bacteria, mycelia and debris. Urine culture grew E Coli and identified Aspergillus Niger.

Enhanced computed tomograms revealed massive gas in the bladder, dissecting in the submucosal layer and extending into the space of Retzius. Debris is outlined by the gas. A shaggy mass engulfes the tip of the Foley catheter. The mass and shaggy debris are caused by a myceliatomas (Figure-1). A CT section at a slightly higher level demonstrated lateral extension of the gas-dissection in the pre-vesical space. It also shows the site of implantation of the transplant ureter, which is edematous (Figure-2). A coronal reconstruction shows the massively edematous ureter, with some gas dissecting into the ureter
(Figure-3). There is however, still some parenchymal phase enhancement of the transplant kidney, suggesting viability.

Open surgical drainage of the space of Retzius was immediately undertaken. Aggressive antibiotic therapy with ciprofloxacin and amphotericin B, parenteral, as well by infusion into the bladder was pursued for 3 weeks. The necrotic transplant ureter was resected. The transplant kidney was drained by percutaneous nephrostomy, ultimately a ureteropyelostomy, using the still intact native ureter was performed to reestablish drainage to the bladder.

Only a few cases of emphysematous cystitis in renal transplant recipients have been reported $(1,2)$.

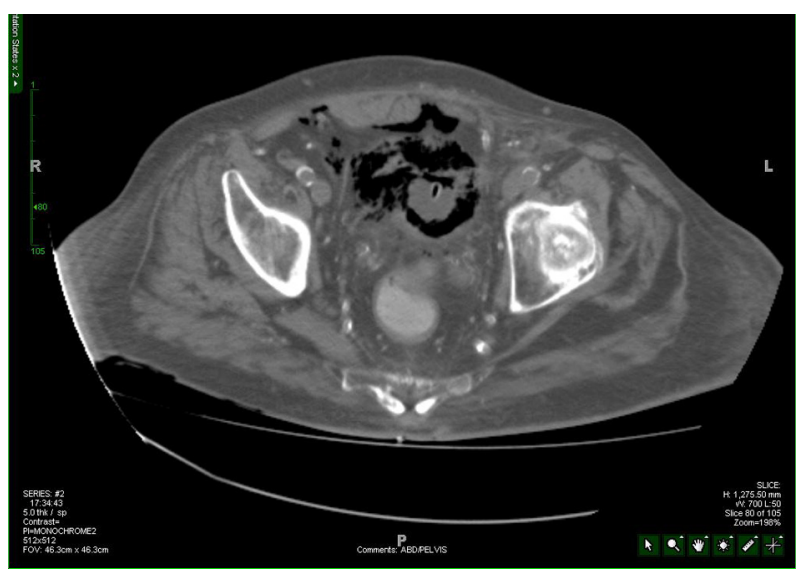

Figure 1 - Massive gas in the bladder, dissecting in the submucosal layer and extending into the space of Retzius. 


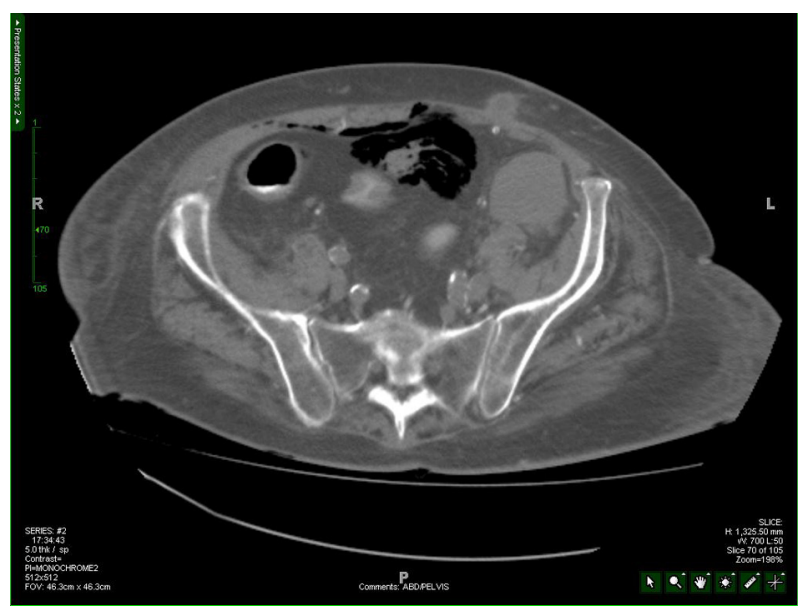

Figure 2 - Lateral extension of the gas-dissection in the prevesical space.

The presence of a gas-forming infection in a patient under maintenance immunosuppression further increases the risk of emphysematous cystitis (mortality up to $20 \%$ ) and calls for prompt diagnosis and aggressive surgical and medical management (1).

\section{REFERENCES}

1. Sampathkumar K, Murali TR, Sooraj YS, Mahaldar AR: Emphysematous prostatitis in renal transplant. Indian J Urol. 2007; 23: 476-8.

\section{Correspondence address:}

Dr. Erich K. Lang

Departments of Urology and Radiology

SUNY, Downstate Health Science Center

455 Lenox Road

Brooklyn, NY, 11203, USA

E-mail: erich.lang@downstate.edu

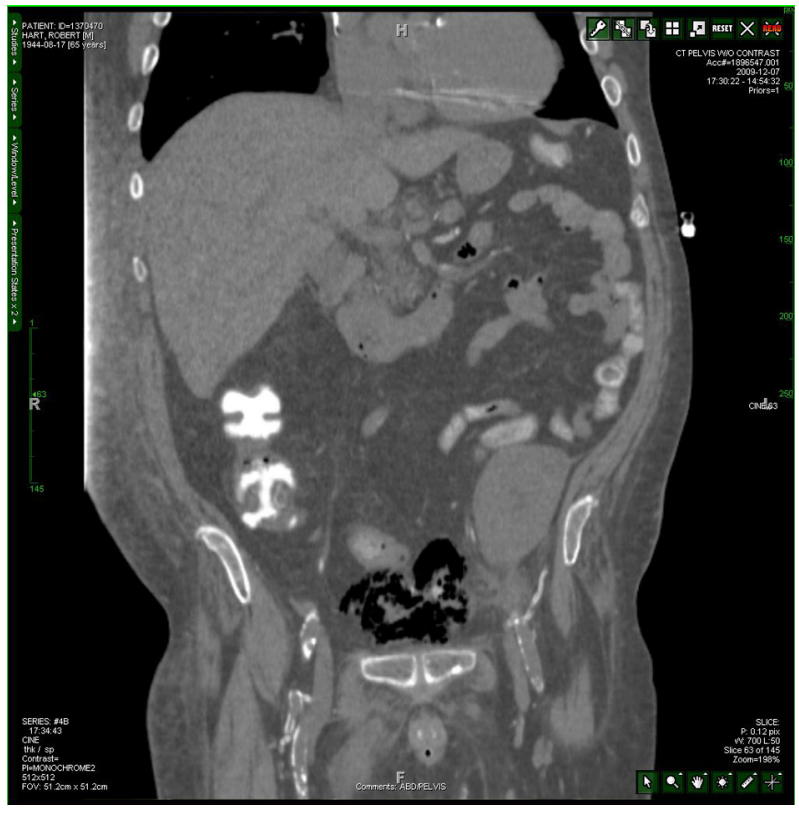

Figure 3 - Gas dissecting into the ureter.

2. Fujita S, Watanabe J, Reed AI, Hemming AW, Solis D, Netzel TC, et al.: Case of emphysematous pyelonephritis in a renal allograft. Clin Transplant. 2005; 19 : 559-62. 